\title{
INVESTIGATING THE “KINK” PLOT AS A SIGNAL OF THE ONSET OF DECONFINEMENT
}

\author{
M. Naskręt ${ }^{\text {a,b }}$ (for the NA61/SHINE collaboration) \\ a Institute of Theoretical Physics, pl. Maxa Borna 9, 50-204 Wrockaw, Poland \\ ${ }^{\mathrm{b}}$ NA61/SHINE collaboration, CERN, Geneva, Switzerland \\ Email: michal.naskret@uwr.edu.pl
}

Received 12 April 2017; revised 20 June 2017; accepted 20 September 2017

\begin{abstract}
One of the physics goals of the NA61/SHINE collaboration at the CERN Super Proton Synchrotron is to study the phase diagram of hadronic matter. To this end, a series of heavy ion collision measurements are performed. It is believed that above a certain collision energy and system size a phase transition between the hadronic matter and quark-gluon plasma occurs. A number of observables have been developed to determine which of the phases was created at the early stage of the collision. This report discusses the dependence of the ratio of the mean number of produced pions to the mean number of wounded nucleons on the Fermi energy measure. For comparison with other measurements this is often presented in the form of the "kink" plot. This plot is presented enriched with preliminary results for ${ }^{40} \mathrm{Ar}+{ }^{45} \mathrm{Sc}$ central collisions at $13 \mathrm{~A}, 19 \mathrm{~A}, 30 \mathrm{~A}, 40 \mathrm{~A}, 75 \mathrm{~A}$ and $150 \mathrm{~A} \mathrm{GeV} / \mathrm{c}$ beam momenta. The results are finally compared to data from other experiments.
\end{abstract}

Keywords: NA61/SHINE, CERN, quark-gluon plasma, onset of deconfinement

PACS: $25.75 .-\mathrm{q}, 25.75 . \mathrm{Nq}$

\section{Introduction}

NA61/SHINE at the CERN Super Proton Synchrotron (SPS) is a fixed target experiment which studies the final hadronic states produced in interactions between various particles at different collision energies [1]. Search for the onset of deconfinement of a strongly interacting matter is part of the physics program of the experiment. Within the framework of this program measurements of $\mathrm{p}+\mathrm{p}, \mathrm{Be}+\mathrm{Be}, \mathrm{p}+\mathrm{Pb}, \mathrm{Ar}+\mathrm{Sc}$ and $\mathrm{Pb}+\mathrm{Pb}$ collisions were performed. In the near future, measurements of $\mathrm{Xe}+\mathrm{La}$ reactions are planned. The measurements will greatly benefit the two-dimensional system size and collision energy scan, which will be helpful in studying the phase transition between hadronic and deconfined phases.
The NA61/SHINE detector (see Fig. 1) is a large-acceptance hadron spectrometer located on the SPS ring at CERN. Upstream from the spectrometer are placed scintillators, Cherenkov detectors and beam position detectors. They provide timing references and position measurements for incoming beam particles. About four meters after the target, the trigger scintillator counter S4 detects if collisions are in the target area by the absence of a beam particle signal. The main detectors of NA61/SHINE are four large volume time projection chambers (TPCs) used for the determination of trajectories and the energy loss $\mathrm{d} E / \mathrm{d} x$ of produced charged particles. The first two - VTPC-1 and VTPC2 - are placed in a magnetic field to measure the particle charge and momentum. Two large TPCs 


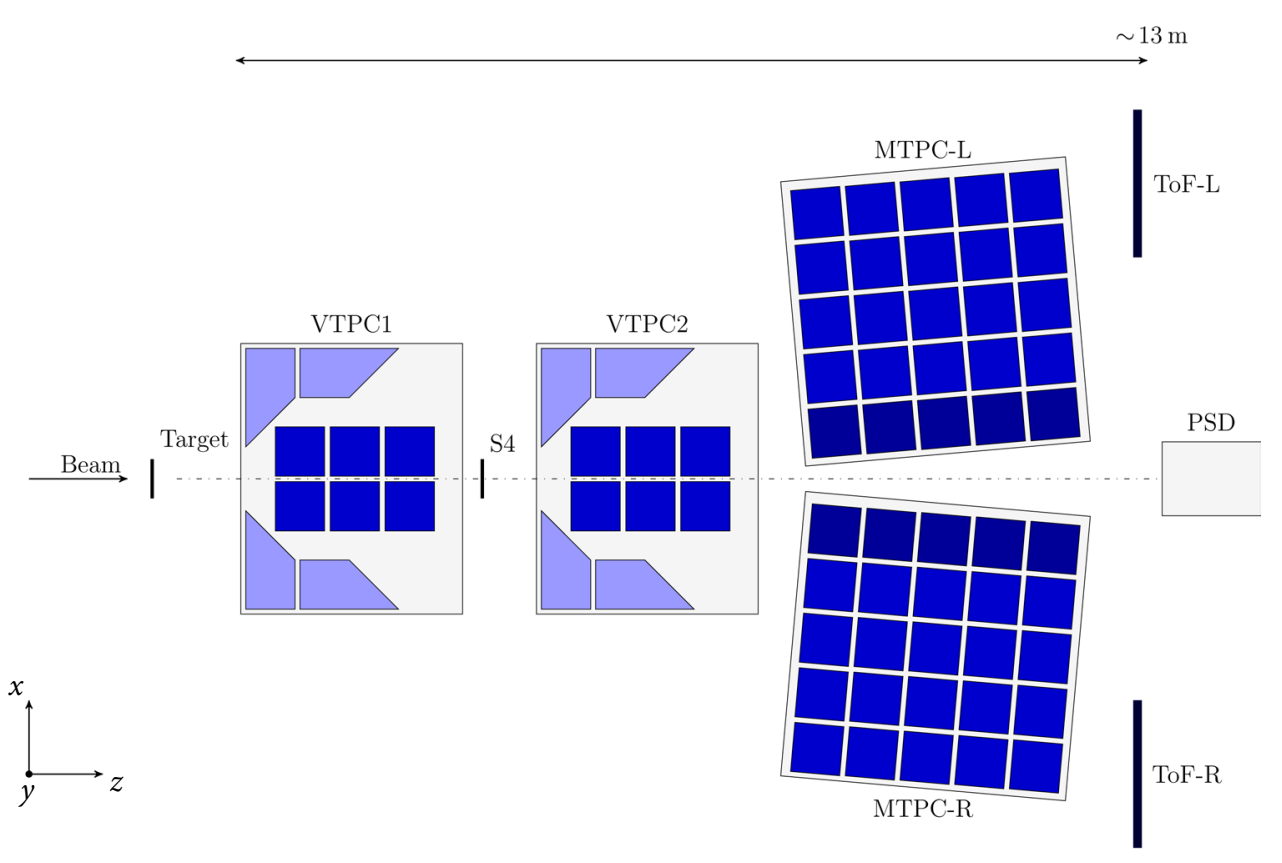

Fig. 1. Experimental setup of NA61/SHINE (coloured online).

(MTPC-L and MTPC-R) are placed downstream of the magnets. Time-of-flight (ToF) measurements are performed by two ToF walls (ToF-L, ToF-R). The last part of the setup is the projectile spectator detector (PSD) - a zero-degree calorimeter which measures the energy of projectile spectators.

\section{The "kink" plot}

According to the Standard Model, quarks and gluons are the elementary constituents of the hadronic matter. Interactions between them are described by quantum chromodynamics [2, 3$]$. One of the most important properties of quarks and gluons is that in the matter under normal conditions they are always enclosed within hadrons. This is called the quark confinement. In order to transform the matter from the hadronic into the deconfined phase one needs to create a system with high temperature and system density conditions. This can be achieved experimentally in ultra-relativistic heavy ion collisions. The system that achieves necessary conditions during the early stage of the collision forms the quark-gluon plasma [4], which rapidly expands and freezesout. In this later stage of the collision, quarks and gluons recombine and form a new set of hadrons.

Since the number of degrees of freedom is higher for the quark-gluon plasma than for the confined matter, it is expected that the entropy density of the system at the given temperature and density should also be higher in the first case. The majority of particles $(\sim 90 \%)$ produced in heavy ion collisions are $\pi$ mesons. Therefore, the entropy and information regarding the state of the matter formed in the early stage of collision should be reflected in the number of produced pions normalized to the volume of the system. This intuitive argument was quantified within the Statistical Model of the Early Stage (SMES) [5]. The change of the mean number of produced pions $\langle\pi\rangle$ is often normalized to the number of wounded nucleons $\langle W\rangle[6]$ and plotted against the Fermi energy measure

$$
F=\left[\left(\sqrt{s_{\mathrm{NN}}}-2 m_{\mathrm{N}}\right)^{3} / \sqrt{s_{\mathrm{NN}}}\right]^{1 / 4},
$$

where $\sqrt{s_{\mathrm{NN}}}$ is centre-of-mass collision energy. As the plot resembles a linear increase with a kink of slope in the case of nucleus-nucleus collisions, it is often referred to as the "kink" plot. The plot is presented in Fig. 5 .

\section{The "kink" plot enriched with preliminary results from Ar+Sc collisions}

In order to add a new point to the "kink" plot it is necessary to calculate the mean number of pions produced in a collision and the mean number of wounded nucleons. The former is extracted from experimental data. In this paper, the preliminary results obtained from the $\mathrm{Ar}+\mathrm{Sc}$ central collisions 
at $13 A, 19 A, 30 A, 40 A, 75 A$ and $150 A \mathrm{GeV} / \mathrm{c}$, taken during the 2015 NA61/SHINE physics data run, are discussed. The number of wounded nucleons is not measured experimentally and has to be calculated using Monte Carlo (MC) simulations.

\subsection{Calculating the mean number of produced pions}

The starting point of the analysis described herein is the double differential spectra $\mathrm{d} n / \mathrm{d} y \mathrm{~d} p_{\mathrm{T}}$ of negatively charged hadrons, where $y$ and $p_{\mathrm{T}}$ are the rapidity and transverse momentum of the hadrons, respectively (see Fig. 2). Centrality was determined by selecting $5 \%$ of collisions with the smallest forward going energy as measured by the projectile spectator detector.

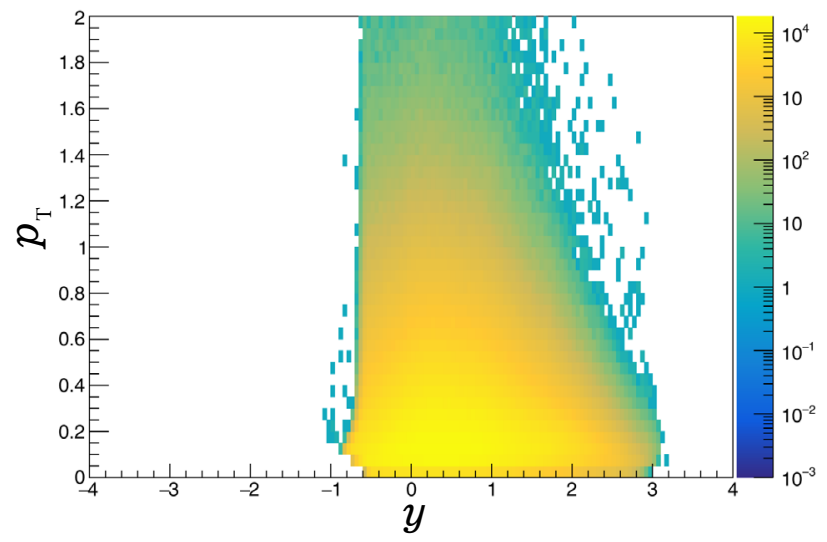

Fig. 2. Example of the double differential spectrum $\mathrm{d} n / \mathrm{d} y \mathrm{~d} p_{\mathrm{T}}$ (coloured online).

The spectra were obtained from the reconstructed tracks applying a series of quality cuts. In order to correct trigger and reconstruction inefficiencies, one needs to apply the Monte Carlo correction. To this end, the EPOS MC [ []] is used in NA61/SHINE. A large statistics of particle collisions is generated and particles are accumulated in bins $n_{\text {gen }}^{i j}$ in the transverse momentum $p_{\mathrm{T}}$ versus rapidity. The generated data undergo a regular reconstruction procedure. Selecting of negatively charged pions results in the distribution $n_{\text {sel }}^{i, j}$. The correction factor $c^{i j}$ is then calculated as the ratio of the two Monte Carlo generated spectra, $c^{i, j}=n_{\text {gen }}^{i, j} / n_{\text {sel }}^{i, j}$ The final experimental spectra are obtained as

$$
n^{i, j}=n_{\text {data }}^{i, j} c^{i, j} .
$$

The NA61/SHINE experimental apparatus is characterized by a large, but limited acceptance. In order to estimate the mean $\pi^{-}$multiplicity in the full acceptance, one needs to extrapolate the experimental data to unmeasured regions. The procedure consists of the following steps:

1 . For the fixed $y$ extrapolate the $p_{\mathrm{T}}$ spectrum from the edge of acceptance to $p_{\mathrm{T}}=2 \mathrm{GeV} / \mathrm{c}$, using the exponential form

$$
f\left(p_{\mathrm{T}}\right)=c p_{\mathrm{T}} \exp \left(\frac{-\sqrt{p_{\mathrm{T}}^{2}+m^{2}}}{T}\right),
$$

where $c$ and $T$ are fitting parameters. To obtain $\mathrm{d} n / \mathrm{d} y$, the measured $p_{\mathrm{T}}$ data bins are summed and the integral of the extrapolated curve is added:

$$
\frac{\mathrm{d} n}{\mathrm{~d} y}=\sum_{0}^{p_{\mathrm{T}}^{\max }} \mathrm{d} p_{\mathrm{T}}\left(\frac{\mathrm{d} n}{\mathrm{~d} y \mathrm{~d} p_{\mathrm{T}}}\right)_{\text {measured }}+\int_{p p_{\mathrm{T}}^{\max }}^{2} f\left(p_{\mathrm{T}}\right) \mathrm{d} p_{\mathrm{T}} .
$$

2. The corrected rapidity spectrum is extrapolated to missing rapidity acceptance, using a sum of two symmetrically displaced Gaussians,

$$
\begin{aligned}
& g(y)=\frac{A_{0} A_{\text {rel }}}{\sigma \sqrt{2 \pi}} \exp \left(-\frac{\left(y-y_{0}\right)^{2}}{2 \sigma^{2}}\right) \\
& +\frac{A_{0}}{\sigma \sqrt{2 \pi}} \exp \left(-\frac{\left(y+y_{0}\right)^{2}}{2 \sigma^{2}}\right)
\end{aligned}
$$

where $A_{0}$ and $A_{\text {rel }}$ are absolute and relative fitted Gaussian amplitudes.

The procedure is presented schematically in Fig. 3 .

The total mean $\pi^{-}$multiplicity is given by the formula

$$
\begin{aligned}
& \left\langle\pi^{-}\right\rangle=\int_{-4}^{y_{\min }} g(y) \mathrm{d} y+\sum_{y_{\min }}^{y_{\max }} \mathrm{d} y\left(\frac{\mathrm{d} n}{\mathrm{~d} y}\right)_{\text {extrapolatedin } p_{\mathrm{T}}} \\
& +\int_{y_{\max }}^{4} g(y) \mathrm{d} y .
\end{aligned}
$$

The results of this procedure are presented in Table 1 . The statistical uncertainties $\sigma_{\text {stat }}\left(\left\langle\pi^{-}\right\rangle\right)$were obtained by propagating the statistical uncertainties of the $\mathrm{d} n / \mathrm{d} y \mathrm{~d} p_{\mathrm{T}}$ spectra. The systematic uncertainties $\sigma_{\text {sys }}\left(\left\langle\pi^{-}\right\rangle\right)$are assumed to be $5 \%$ based on the previous NA61 analysis of $\mathrm{p}+\mathrm{p}$ collisions in [8]. The main sources of the latter uncertainties are possible biases 

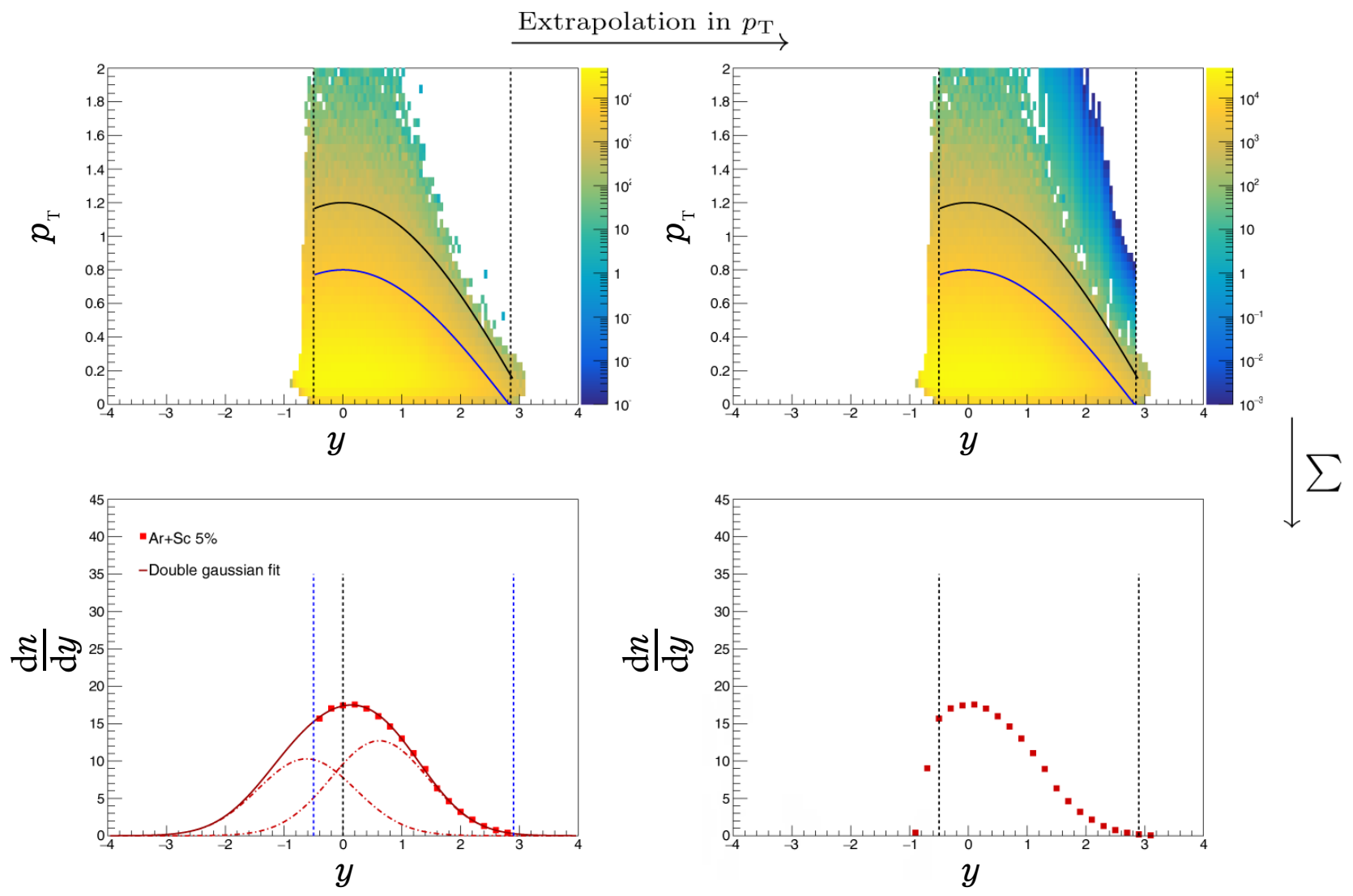

$\stackrel{\text { Fitting Gaussians }}{\longleftarrow}$

Fig. 3. Scheme of the extrapolation procedure (coloured online).

Table 1. Mean $\pi^{-}$multiplicities in the $5 \%$ most central $\mathrm{Ar}+\mathrm{Sc}$ collisions with systematic and statistical uncertainties.

\begin{tabular}{ccc|c|c|c|c|c}
\hline Momentum $[A \mathrm{GeV} / c]$ & 13 & 19 & 30 & 40 & 75 & 150 \\
\hline$\left\langle\pi^{-}\right\rangle$ & 38.46 & 48.03 & 59.72 & 66.28 & 86.12 & 108.92 \\
\hline$\sigma_{\text {stat }}\left(\left\langle\pi^{-}\right\rangle\right)$ & \pm 0.021 & \pm 0.021 & \pm 0.024 & \pm 0.018 & \pm 0.0079 & \pm 0.0088 \\
\hline$\sigma_{\text {stat }}\left(\left\langle\pi^{-}\right\rangle\right)$ & \pm 1.92 & \pm 2.40 & \pm 2.98 & \pm 3.31 & \pm 4.30 & \pm 5.44 \\
\hline
\end{tabular}

from the event and track selection, the uncertainty of the Monte Carlo correction factor, and the uncertainty due to the track selection method.

\subsection{Calculating the mean number of wounded nucleons}

The number of wounded nucleons cannot be measured experimentally in NA61/SHINE. It has to be calculated using Monte Carlo models. Two models were used to perform calculations - Glissando 2.73 [9] based on the Glauber model and EPOS 1.99 (version CRMC 1.5.3) [7] using the parton ladder model. A procedure to obtain a reliable number from the models was developed. Glissando provides a value that is consistent with previous measurements and applicable to the wounded nucleon model [6]. EPOS, on the other hand, allows a more detailed centrality analysis and event selection. It is possible to reproduce Glauber-based values in EPOS and they are in good agreement with Glissando as shown in Fig. 4 .

In order to estimate systematic uncertainties connected with the calculation of $\langle W\rangle$, the impact of the cross section uncertainties and centrality determination uncertainty was assessed. The former was calculated propagating the cross 


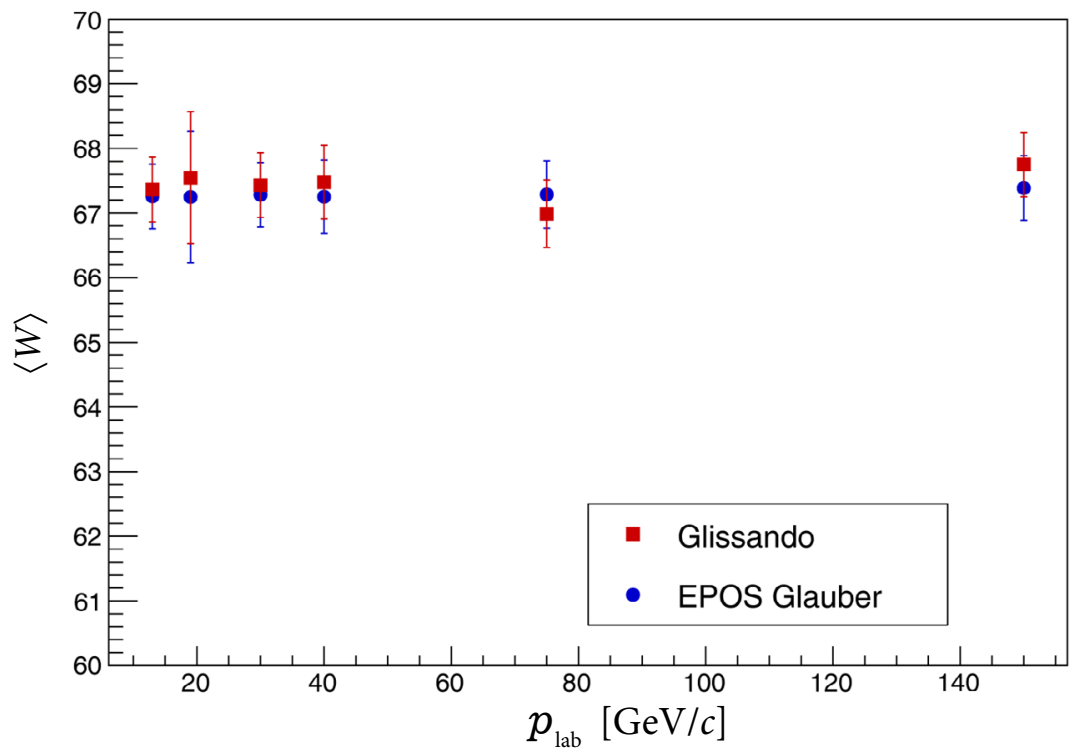

Fig. 4. Comparison of Glissando and EPOS "a la Glauber" values of $\langle W\rangle$ (coloured online).

Table 2. $\langle W\rangle$ in the 5\% most central Ar+Sc collisions calculated by EPOS together with the statistical $\sigma_{\text {stat }}$ and systematic $\sigma_{\mathrm{sys}}$ uncertainty expressed as percentage of $\langle W\rangle$.

\begin{tabular}{cccccccc}
\hline Momentum $[A \mathrm{GeV} / c]$ & 13 & 19 & 30 & 40 & 75 & 150 \\
\hline$\langle W\rangle$ & 67.26 & 67.25 & 67.28 & 67.25 & 67.29 & 67.39 \\
\hline$\sigma_{\text {stat }}(\langle W\rangle)$ & $0.0069 \%$ & $0.0069 \%$ & $0.0071 \%$ & $0.0069 \%$ & $0.0069 \%$ & $0.0071 \%$ \\
\hline$\sigma_{\text {sys }}(\langle W\rangle)$ & $0.74 \%$ & $1.52 \%$ & $0.75 \%$ & $0.84 \%$ & $0.77 \%$ & $0.75 \%$ \\
\hline
\end{tabular}

section uncertainties [10] to Monte Carlo models. The centrality determination in calculating $\langle W\rangle$ is based on the number of projectile spectators which can be chosen only as integer numbers and will therefore have a contribution to the systematic uncertainty. The estimated values of the systematic uncertainty together with the statistical uncertainty are presented in Table 2 .

\section{Conclusions}

As for NA61/SHINE there are only results for $\left\langle\pi^{-}\right\rangle$ in $\mathrm{Ar}+\mathrm{Sc}$ collisions, the pion multiplicity was approximated by $\langle\pi\rangle_{\mathrm{Ar}+\mathrm{Sc}}=3\left\langle\pi^{-}\right\rangle_{\mathrm{Ar}+\mathrm{Sc}}$. This allows one to produce a preliminary version of the "kink" plot shown in Fig. 5 .

From the preliminary version of the "kink" plot one can conclude that for high SPS energies $\mathrm{Ar}+\mathrm{Sc}$ measurements lie in the $\mathrm{Pb}+\mathrm{Pb}$ region. On the other hand, for low SPS energies Ar+Sc measurements lie in the $\mathrm{p}+\mathrm{p}$ region. This intermediate collision system does not behave as a lighter system or as a heavier one. For lower SPS energies the pion production is enhanced compared to heavier systems, whereas for high SPS energies it is enhanced com- pared to lighter ones. The intermediate energies show the cross-over behaviour. This observation might indicate that in the system size scan, $\mathrm{Ar}+\mathrm{Sc}$ is the first one to experience effects such as phase transition between the confined and deconfined

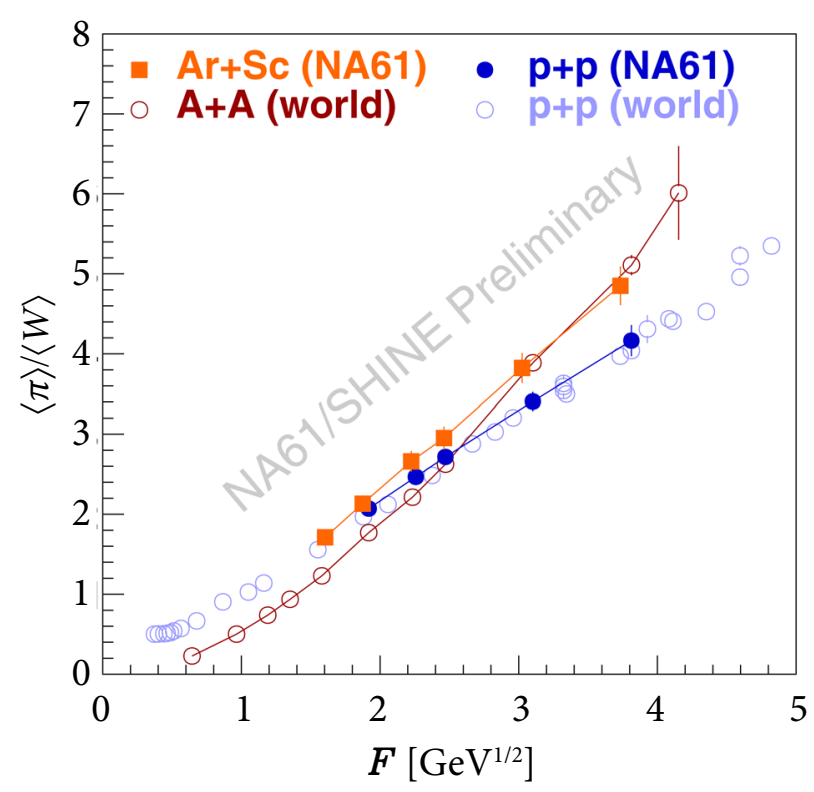

Fig. 5. The "kink" plot with new measurements (coloured online). 
matter or the percolation threshold [11]. A further investigation of the preliminary results and improved centrality selection is urgently needed.

\section{Acknowledgements}

This work was partially supported by the National Science Centre of Poland grant Harmonia 7 2015/18/M/ST2/00125.

\section{References}

[1] N. Abgrall, O. Andreeva, A. Aduszkiewicz, Y. Ali, T. Anticic, N. Antoniou, B. Baatar, F. Bay, A. Blondel, J. Blumer, et al., NA61/SHINE facility at the CERN SPS: beams and detector system, J. Instrum. 9, P06005 (2014).

[2] M. Gell-Mann, A schematic model of baryons and mesons, Phys. Lett. 8, 214-215 (1964).

[3] G. Zweig, An SU(3) Model for Strong Interaction Symmetry and Its Breaking, CERN Report No. 8182/TH.401 (1964).

[4] J.C. Collins and M.J. Perry, Superdense matter: Neutrons or asymptotically free quarks? Phys. Rev. Lett. 34, 1353 (1975).

[5] M. Gaździcki and M.I. Gorenstein, On the early stage of nucleus-nucleus collisions, Acta Phys. Pol. B 30, 2705-2735 (1999).
[6] A. Białas, M. Bleszyński, and W. Czyż, Multiplicity distributions in nucleus-nucleus collisions at high energies, Nucl. Phys. B 111(3), 461-476 (1976).

[7] K. Werner, F.-M. Liu, and T. Pierog, Parton ladder splitting and the rapidity dependence of transverse momentum spectra in deuterongold collisions at the BNL Relativistic Heavy Ion Collider, Phys. Rev. C 74, 044902 (2006).

[8] N. Abgrall, A. Aduszkiewicz, Y. Ali, T. Anticic, N. Antoniou, B. Baatar, F. Bay, A. Blondel, J. Blumer, M. Bogomilov, et al., Measurement of negatively charged pion spectra in inelastic $\mathrm{p}+\mathrm{p}$ interactions at $p_{\mathrm{lab}}=20,31,40,80$ and $158 \mathrm{GeV} / c$, Eur. Phys. J. C 74(3), 2794 (2014).

[9] M. Rybczyński, G. Stefanek, W. Broniowski, and P. Bozek, GLISSANDO 2: GLauber Initial-State Simulation AND mOre..., ver. 2, Comput. Phys. Commun. 185(6), 1759-1772 (2014).

[10]C. Patrignani, K. Agashe, G. Aielli, C. Amsler, M. Antonelli, D.M. Asner, H. Baer, S. Banerjee, R.M. Barnett, T. Basaglia, et al. (Particle Data Group), Review of Particle Physics, Chin. Phys. C 40(10), 100001 (2016).

[11]G. Baym, Confinement of quarks in nuclear matter, Phys. A 96(1), 131-135 (1979).

\title{
„PŪPSNIO“ GRAFIKO KAIP SIGNALO APIE KVARKŲ IŠLAISVĖJIMO PRADŽIĄ TYRIMAS
}

\author{
M. Naskręt ${ }^{\mathrm{a}, \mathrm{b}}$ \\ a Teorines fizikos institutas, Vroclavas, Lenkija \\ ${ }^{\mathrm{b}}$ NA61/SHINE sambūris, CERN, Ženeva, Šveicarija
}

